Bundesgesundheitsbl 2019 · 62:652-661 https://doi.org/10.1007/s00103-019-02940-y Online publiziert: 23. April 2019

(c) Der/die Autor(en) 2019

Fachübergreifender Arbeitskreis „Bundesweites Pollenmonitoring“

Berlin, Deutschland

\title{
Perspektiven für ein bundesweites Pollenmonitoring in Deutschland
}

„Umweltmedizin und gesundheitliche Bewertung", 23 Einrichtungen und Institutionen zur Mitarbeit in einem fachübergreifenden Arbeitskreis „Bundesweites Pollenmonitoring" eingeladen. Die Auswahl der Einrichtungen und Institutionen war in Zusammenarbeit mit externen Fachleuten erfolgt, Kriterien waren veröffentlichte Arbeiten zum Thema Pollenmonitoring und/oder öffentlich bekanntes bzw. angenommenes Interesse am Thema Pollenmonitoring. Sechzehn Einrichtungen und Institutionen nahmen die Einladung an (- Infobox 1). Alle im Arbeitskreis mitarbeitenden Einrichtungen und Institutionen waren gleichberechtigt vertreten. Das Umweltbundesamt, Fachgebiet II 1.5, übernahm über seine Mitarbeit hinaus die Funktion einer Geschäftsstelle.

Zwischen November 2017 und Juni 2018 kam der Arbeitskreis zu insgesamt drei Gesprächstagen zusammen, um sich über Perspektiven für ein bundesweites Pollenmonitoring auszutauschen. Die jeweiligen Themenschwerpunkte der Gesprächstage sind in $\bullet$ Tab. 1 zusammengefasst.

Für Gesprächstag 2 hatte der Arbeitskreis internationale Gäste eingeladen:

- Uwe Berger, Medizinische Universität Wien, Forschungsgruppe Aerobiologie und Polleninformation, Österreich,

- Yolanda Clewlow, Met Office, Großbritannien,

- Bernard Clot, MeteoSchweiz, Schweiz,

- Mikhail Sofiev, Finnisches Meteorologisches Institut, Finnland,
- Michel Thibaudon, Französisches Aerobiologisches Netzwerk, Frankreich.

Für Gesprächstag 3 wurden nationale Gäste eingeladen:

- Ute Dauert, Umweltbundesamt, Fachgebiet II 4.2, „Beurteilung der Luftqualität",

- Jörg Rechenberg, Umweltbundesamt, Fachgebiet II 2.1, „Übergreifende Angelegenheiten Wasser und Boden“, - Marc Röckinghausen, Fachhochschule für öffentliche Verwaltung Nordrhein-Westfalen.

Für jeden der drei Gesprächstage wurde ein für den internen Gebrauch bestimmtes und mit allen Mitgliedern des Arbeitskreises abgestimmtes Protokoll erstellt. Die Protokolle der Gesprächstage bildeten anschließend die Grundlage für die Erstellung dieses Positionspapiers, mit dem der Arbeitskreis seine Arbeit abschließt.

Für eine erste Vorstellung der Position des Arbeitskreises in der 21. Sitzung der Länderarbeitsgruppe Umweltbezogener Gesundheitsschutz (LAUG) vom 25.09.2018 bis 27.09.2018 in Kiel hatte der Arbeitskreis wesentliche Inhalte der Gesprächstage vorab in einem Kurzpapier zusammengefasst. Es kann beim Arbeitskreis angefordert werden.

\section{Ergebnisse}

\section{Pollendaten - für wen und wozu?}

\section{Gesundheitliche Relevanz von Pollen}

Pollen sind luftgetragene Partikel biologischer Herkunft [2]. Allergene Pollen sind 


\section{Infobox 1 : Im fachübergreifen- den Arbeitskreis „Bundesweites Pollenmonitoring" vertretene Einrichtungen und Institutionen in alphabetischer Reihenfolge}

Der Arbeitskreis formierte sich im Sommer 2017 und beendet seine Arbeit mit der Veröffentlichung dieses Positionspapiers.

- Ärzteverband Deutscher Allergologen e.V. (AeDA)

- Bayerisches Landesamt für Gesundheit und Lebensmittelsicherheit (LGL), AP 2 Arbeits- und Umweltmedizin/ Epidemiologie

- Bund/Länder-Arbeitsgemeinschaft Immissionsschutz (LAl), Ausschuss Luftqualität/Wirkungsfragen/Verkehr (LWV)

- Deutsche Gesellschaft für Allergologie und klinische Immunologie (DGAKI)

- Deutscher Allergie- und Asthmabund e.V. (DAAB)

- Deutscher Wetterdienst (DWD), Referat Lufthygiene

- Freie Universität Berlin (FU Berlin), Institut für Meteorologie

- Gesellschaft für Hygiene, Umweltmedizin und Präventivmedizin (GHUP)

- Landesamt für Gesundheit und Soziales Mecklenburg-Vorpommern (LAGuS M-V), Abteilung 3, Fachbereich Infektiologie

- Länderarbeitsgruppe Umweltbezogener Gesundheitsschutz (LAUG)

- Ministerium für Arbeit, Soziales, Gesundheit, Frauen und Familie Brandenburg (MASGF), Referat 43, Öffentlicher Gesundheitsdienst, Infektionsschutz, Umwelthygiene, Zivil- und Katastrophenschutz

- Rheinisch-Westfälische Technische Hochschule Aachen (RWTH Aachen), Klinik für Dermatologie und Allergologie

- Stiftung Deutscher Polleninformationsdienst (PID)

- Umweltbundesamt (UBA), Abteilung II 4, "Luft"

- Umweltbundesamt (UBA), Fachgebiet II 1.5, „Umweltmedizin und gesundheitliche Bewertung"

- Verein Deutscher Ingenieure (VDI e.V.), VDI/DIN-Kommission Reinhaltung der Luft (KRdL) - Normenausschuss, Richtlinienausschuss NA 134-03-07-10 UA „Erfassen allergierelevanter luftgetragener Pollen"

- Zentrum Allergie und Umwelt (ZAUM), Technische Universität München und Helmholtz Zentrum München der Hauptauslöser der Atemwegserkrankungen allergische Rhinitis (Synonyme: Heuschnupfen, Heufieber, Pollinosis) und allergisches Asthma [3, 4]. Zu den pollenassoziierten allergischen Erkrankungen gehört darüber hinaus das sogenannte orale Allergiesyndrom (OAS, Synonyme: pollenassoziierte Nahrungsmittelallergie, Nahrungsmittel-PollenSyndrom; [4]).

In Deutschland erkranken derzeit ${ }^{2}$ $15 \%$ der Erwachsenen und $11 \%$ der Kinder mindestens einmal in ihrem Leben an einer allergischen Rhinitis (sogenannte Lebenszeitprävalenz) - das entspricht etwa 11 Mio. betroffenen Menschen; 9\% der Erwachsenen bzw. 5\% der Kinder erkranken an Asthma [3, 5, 6]. Bei Nahrungsmittelallergien, von denen bei Erwachsenen ein Großteil auf Sensibilisierungen gegen Pollenallergene beruht (siehe OAS), liegt die Lebenszeitprävalenz für Erwachsene derzeit bei $5 \%[5,7]$.

Noch höher als die Zahl der Erkrankten liegt die Zahl der gegen Pollenallergene sensibilisierten Menschen: So sind in Deutschland derzeit 18\% der Erwachsenen gegen Gräserpollen und $17 \%$ gegen Birkenpollen sensibilisiert [8].

Eine Zunahme der Exposition gegenüber allergenen Pollen führt $\mathrm{zu}$ einer Zunahme der Sensibilisierungen, der Erkrankungen und der Beschwerden [9-13].

\section{Kosten pollenassoziierter allergischer Erkrankungen}

Pollenassoziierte allergische Erkrankungen verursachen dem Gesundheitssystem und der Gesellschaft jährliche Kosten im mehrstelligen Millionen- bis Milliardenbereich [14-16]. Für saisonale allergische Rhinitis wurden die Kosten pro Patient und Jahr für Deutschland auf $1543 €$ beziffert, für allergisches Asthma auf $9286 €$ (Berechnungen für den Zeitraum 1999/2000, nur Erwachsene; [15]). Für Bayern liegen Berechnungen des Bayerischen Landesamtes für Gesundheit und Lebensmittelsicherheit (LGL) für das Jahr 2013 vor [16]: Danach

\footnotetext{
2 Zeitraum der Datenerhebung: Erwachsene 2008-2011, Kinder 2003-2006, Details siehe [5, $6]$.
}

verursachten Pollenallergien dem Gesundheitssystem und der Gesellschaft im Jahr 2013 Kosten in Höhe von 609 Mio. $€$. Etwa die Hälfte davon ließ sich direkten Krankheitskosten zuordnen, wie z.B. Kosten für Medikamente und Krankenhausaufenthalte, die andere Hälfte indirekten Krankheitskosten z. B. durch Arbeitsunfähigkeit oder verminderte Arbeitsleistung.

\section{Gesundheitlicher Nutzen von Pollendaten}

Allergiebedingte Krankheitskosten würden sich durch bessere Versorgungsstrukturen deutlich senken lassen [14]. Im Falle pollenassoziierter allergischer Erkrankungen schließt dies nach Meinung von Experten die freie Verfügbarkeit repräsentativer (Echtzeit-)Pollendaten und Pollenflugvorhersagen ein [17].

Pollendaten sind wesentlicher Bestandteil der gesundheitlichen Vorsorge:

- Sie gehen in die Pollenflugvorhersage ein (Tertiärprävention: Anpassung von Verhalten und Medikamenteneinnahme; [4]).

- Sie stellen ein diagnostisches Hilfsmittel für Ärzte dar (Sekundärprävention: Diagnose einer Pollenallergie, Management der spezifischen Immuntherapie, Kontrolle der Wirksamkeit der spezifischen Immuntherapie; [4]).

- Sie zeigen das Auftreten neuer Pollen an (Primärprävention: ggf. Verhinderung der Pollenausbreitung und damit der allergischen Sensibilisierung; [18]).

Die Messung allergener Pollen ermöglicht darüber hinaus das Erkennen von Veränderungen im Pollenspektrum, wie sie z.B. im Rahmen des Klimawandels oder veränderter Flächennutzungen zu erwarten sind [19, 20]. Das Wissen um Veränderungen im Pollenspektrum ist Voraussetzung für Anpassungen in der gesundheitlichen Vorsorge (z.B. Entwicklung neuer Diagnostika und Therapeutika). 


\section{Nutzen von Pollendaten: Weitere Anwendungsbereiche}

Über den gesundheitlichen Nutzen hinaus finden Pollendaten in verschiedenen anderen Bereichen Anwendung, so z. B. in der Landwirtschaft, der Klimatologie und der Biodiversitätsforschung.

Zusammenfassend profitieren vier Gesellschaftsgruppen bzw. -bereiche von Pollendaten: Patienten, Ärzte, Wissenschaft und Forschung sowie Gesundheitssystem und Volkswirtschaft (- Abb. 1). Die Gruppen bzw. Bereiche bedingen sich gegenseitig. So profitieren Patienten von optimalen Arbeitsbedingungen für Ärzte und durch adäquate Therapien und Verhaltensanpassungen lassen sich direkte und indirekte Krankheitskosten senken.

\section{Methoden zur Erhebung von Pollendaten}

Zur Messung von Pollen in der Außenluft stehen bis dato im Wesentlichen zwei Methodenprinzipien zur Verfügung, passive und aktive volumetrische.

Passive Methoden beruhen auf der Sedimentation von Pollen. Sedimentierende Pollen werden auf beschichteten Flächen gesammelt und anschließend durch Pollenanalysten mikroskopisch untersucht und ausgezählt. Geräte, die auf der Basis dieser Methodik arbeiten, werden als Passivsammler bezeichnet, ein gängiger Vertreter ist z. B. die DurhamFalle [21]. Passivsammler sind preiswert in der Herstellung und leicht handhabbar. Diesen Vorteilen stehen folgende Nachteile gegenüber: (a) Bias zu schwereren Pollen, (b) geringe Vergleichbarkeit zwischen verschiedenen Gerätevertretern, (c) keine volumenbezogenen Messungen und (d) keine Echtzeitmessungen. Aufgrund der Vorteile wurden und werden Passivsammler weltweit vor allem in Studien eingesetzt [22]. In Pollenmessnetzen zur routinemäßigen Erfassung der Hintergrundbelastung finden sie aufgrund der erwähnten Nachteile kaum Verwendung [23].

Aktive volumetrische Methoden beruhen auf dem aktiven Ansaugen eines definierten Luftvolumens und der Impaktion, d.h. der Abscheidung, von Pollen aus dem Ansaugvolumen. Aufgrund

Bundesgesundheitsbl 2019 · 62:652-661 https://doi.org/10.1007/s00103-019-02940-y

(c) Der/die Autor(en) 2019

Fachübergreifender Arbeitskreis „Bundesweites Pollenmonitoring“

\section{Perspektiven für ein bundesweites Pollenmonitoring in Deutschland}

\section{Zusammenfassung}

Allergene Pollen sind natürliche, biologische luftgetragene Partikel und der Hauptauslöser allergischer Atemwegserkrankungen. Die Messung allergener Pollen ist Voraussetzung für die gesundheitliche Vorsorge und zeigt Veränderungen im Pollenspektrum an, wie sie z. B. im Rahmen des Klimawandels zu erwarten sind.

Das in Deutschland einzige bundesweite Pollenmessnetz wird durch die Stiftung Deutscher Polleninformationsdienst betrieben. Fortbestand und Weiterentwicklung (u. a. Hybridsystem aus manuellen und automatischen Pollenfallen) dieses Messnetzes sind finanziell jedoch nicht gesichert. Im Sommer 2017 formierte sich daher der fachübergreifende Arbeitskreis "Bundesweites Pollenmonitoring", um sich über diesbezügliche Perspektiven für Deutschland auszutauschen.

Für den Austausch kam der Arbeitskreis zwischen November 2017 und Juni 2018 zu drei Gesprächstagen mit jeweils unterschiedlichen Themenschwerpunkten zusammen. Die internen Protokolle zu den Gesprächstagen bildeten anschließend die
Grundlage für die Erstellung des vorliegenden Positionspapiers, mit dem der Arbeitskreis seine Arbeit abschließt.

Aufgrund der Bedeutung allergener Pollen für die menschliche Gesundheit und allergischer Erkrankungen für das Gesundheitssystem spricht sich der Arbeitskreis dafür aus, das bundesweite Pollenmonitoring in den Katalog staatlicher Aufgaben aufzunehmen, die der grundlegenden Versorgung der Bevölkerung mit wesentlichen Gütern und Dienstleistungen dienen (öffentliche Daseinsvorsorge).

Hinsichtlich möglicher Zuständigkeiten im Rahmen der öffentlichen Daseinsvorsorge wurden im Arbeitskreis mehrere Lösungsansätze diskutiert. Unabhängig von der zukünftigen Zuständigkeit kommt der Kooperation von messtechnischen, klinischen und wissenschaftlichen Einrichtungen eine grundsätzliche Bedeutung für die adäquate gesundheitliche Vorsorge zu.

Schlüsselwörter

Messung · Vorhersage · Gesundheit · Allergie · Prävention

\section{Perspectives for nationwide pollen monitoring in Germany}

\section{Abstract}

Allergenic pollen are natural, biological airborne particles and the main cause of allergic respiratory diseases. The measurement of allergenic pollen is a required measure in preventive healthcare and indicates changes in the pollen spectrum as to be expected in the context of climate change.

The only nationwide pollen measuring network in Germany is operated by the Foundation German Pollen Information Service, however, its continuity and further development (a hybrid system with manual and automated pollen traps) are not financially guaranteed. In the summer of 2017, the interdisciplinary working group "National Pollen Monitoring" was formed to exchange corresponding perspectives for Germany.

Between November 2017 and June 2018, the working group met for three days of discussions, each with a different topic. The internal protocols for each meeting served as a basis for the position paper, which the working group used to complete their work. Due to the impact of allergenic pollen on human health and the significance of allergic diseases for the health service, the working group is in favour of the nationwide pollen monitoring being included in the catalogue of government responsibilities, which secure the basic supply of essential goods and public services of general interest.

Regarding any possible assigned responsibilities within the governmental framework, different options were discussed. Independent from future responsibilities, cooperation between metrological, medical and scientific facilities is essential for adequate preventive healthcare.

Keywords Measurement $\cdot$ Forecast $\cdot$ Health $\cdot$ Allergy . Prevention 
Tab. 1 Themenschwerpunkte der 3 Gesprächstage des fachübergreifenden Arbeitskreises „Bundesweites Pollenmonitoring

\section{Schwerpunkte}

\begin{tabular}{|ll}
\hline Gesprächstag 1 & $\begin{array}{l}\text { Darstellung und Diskussion der gesundheitlichen Bedeutung eines bundes- } \\
\text { weiten Pollenmessnetzes } \\
\text { Darstellung des Status quo des bundesweiten Pollenmessnetzes } \\
\text { Diskussion und Definition von Minimal- und Idealanforderungen an ein } \\
\text { bundesweites Pollenmessnetz einschließlich der damit verbundenen Kos- } \\
\text { ten pro Jahr }\end{array}$ \\
\hline Gesprächstag 2 & $\begin{array}{l}\text { Pollenmessnetze anderer europäischer Staaten: Finnland, Frankreich, Groß- } \\
\text { britannien, Österreich, Schweiz } \\
\text { Vernetzungen zum Thema Pollenmonitoring auf europäischer Ebene }\end{array}$ \\
\hline Gesprächstag 3 & $\begin{array}{l}\text { Darstellung der gesetzlichen Rahmenbedingungen und Verwaltungsstruk- } \\
\text { turen in Deutschland (Bund- und Länderverantwortlichkeiten) } \\
\text { Erarbeitung von Möglichkeiten eines verlässlichen bundesweiten Pollen- } \\
\text { messnetzes } \\
\text { Pro- und Kontraworkshop }\end{array}$ \\
\hline Die Gesprächstage fanden zwischen November 2017 und Juni 2018 statt
\end{tabular}

ihrer aktuellen Relevanz werden im Folgenden volumetrische Geräte nach dem Hirst-Prinzip und volumetrische Geräte mit automatischer Pollenanalyse detaillierter vorgestellt.

Bei volumetrischen Geräten nach dem Hirst-Prinzip, sogenannten Hirst-TypFallen, wird ein definierter Luftstrom (101/min, entspricht in etwa dem Atemminutenvolumen eines erwachsenen Menschen) über eine Fangtrommel auf eine haftende Folie geführt. Die beiden seit vielen Jahren kommerziell erhältlichen Vertreter dieses Gerätetyps sind die sogenannte Burkard- und die LanzoniFalle, jeweils benannt nach den Namen der Herstellerfirmen [24-26]. Nach der manuellen Präparation der Folie erfolgt die lichtmikroskopische Analyse der Foliensegmente durch Pollenanalysten. Die fachgerechte Pollenmessung mit HirstTyp-Fallen ist in der Richtlinie VDI 4252 Blatt 4 sowie in Kürze in dem auf europäischer Ebene erarbeiteten Standard DIN EN 16868 festgeschrieben [2, 27]. Der Zeitaufwand für die Präparation einer Folie beträgt ca. $45-60 \mathrm{~min}$, der Zeitaufwand für die Auszählung von 7 Pollenarten pro Tag ca. 20-30 min. Damit sind die Pollendaten eines Tages frühestens am Vormittag des Folgetages verfügbar. Theoretisch wäre ein mehrmaliges Präparieren und Auswerten pro Tag möglich, aus Zeit- und Kostengründen ist dieses Herangehen aber kaum praktizierbar.

Volumetrische Geräte mit automatischer Pollenanalyse, sogenannte Pollen- vollautomaten, sind seit einigen Jahren kommerziell erhältlich. Sie lassen sich nach der Analysemethodik unterscheiden in Geräte mit Echtzeitlasermessung und Geräte, die mit Bilderkennungssoftware arbeiten [28]. Pollenvollautomaten bieten gegenüber den volumetrischen Geräten nach dem Hirst-Prinzip vor allem den Vorteil der Echtzeitmessung von Pollen (Wegfall der zeitaufwendigen manuellen Präparation und Analyse), bisherige Nachteile sind die fehlende Standardisierung und Analysefehler (Größenordnung vergleichbar mit dem von Hirst-Typ-Fallen; [29, 30]). In Deutschland sind derzeit vor allem zwei Geräte in den Fokus des Interesses gerückt, der Pollenvollautomat BAA 500, der mit Bilderkennungssoftware arbeitet [31], und der Pollenvollautomat Rapid-E, dessen Messprinzip auf Echtzeitlasermessung beruht [32]. Der BAA 500 kommt im Elektronischen Polleninformationsnetzwerk Bayern (ePIN) zum Einsatz, der Rapid-E wird derzeit vom Deutschen Wetterdienst (DWD) getestet (Details siehe unten).

Um dem Problem der fehlenden Standardisierung von Pollenvollautomaten und damit der fehlenden Vergleichbarkeit von Automatendaten frühzeitig begegnen zu können, hat sich im Jahr 2017 innerhalb des „European Meteorological Services Network" (EUMETNET), einem europäischen Interessen- und Dachverband, dem gegenwärtig 26 nationale Wetterwarndienste angehören, das Programm „AutoPollen“ formiert
[33], in dem auch der DWD, das LGL und das Zentrum Allergie und Umwelt (ZAUM), Technische Universität München und Helmholtz Zentrum München, mitarbeiten.

Hinsichtlich der Methodenstandardisierung fehlt für Pollenmessungen unabhängig von der verwendeten Methodik bisher ein sogenannter Goldstandard, d.h. die Möglichkeit des Mitführens von Luftproben definierter Pollenkonzentration. Dadurch lassen sich Geräte derzeit nur gegeneinander, nicht aber gegen Standardproben vergleichen. Möglicherweise werden Allergenexpositionskammern dieses Problem lösen können [34].

\section{Status quo des bundesweiten Pollenmessnetzes}

\section{Pollenmessungen durch die Stiftung Deutscher Polleninformationsdienst}

Die Stiftung Deutscher Polleninformationsdienst (PID) wurde 1983 auf Initiative von Viktor Ruppert (Ärzteverband Deutscher Allergologen e. V.) gegründet. Stifterin war die Fisons Arzneimittel $\mathrm{GmbH}$, heute Sanofi Aventis. Sitz der Geschäftsstelle ist seit 2009 Berlin. Entsprechend der Satzung ist die Stiftung ,zur Förderung der Gesundheit durch Einrichtung eines bundesweit tätigen und für jedermann erreichbaren Polleninformationsdienstes" verpflichtet [35].

Einer der Hauptschwerpunkte der Stiftung liegt seit ihrer Gründung in der bundesweiten Messung von Pollen in der Außenluft. Das durch die Stiftung aufgebaute Messnetz dient der Erfassung der sogenannten Hintergrundbelastung, in die sowohl der regionale als auch der überregionale (Stichwort Ferntransport) Pollenflug eingehen. Das Messnetz besteht derzeit aus über 30 Messstationen, die in unterschiedlicher Dichte über das Bundesgebiet verteilt sind und ganzjährig (sogenannte Referenzstationen) bzw. saisonal die Pollenbelastung in der AuBenluft messen [36]. Die Stationen sind überwiegend an medizinischen Einrichtungen bzw. Kliniken angesiedelt. Die Standortwahl richtet sich nach dem Ziel der möglichst repräsentativen Erfassung des Pollenfluges für eine gegebene Region und dem Vorhandensein qualitativ 


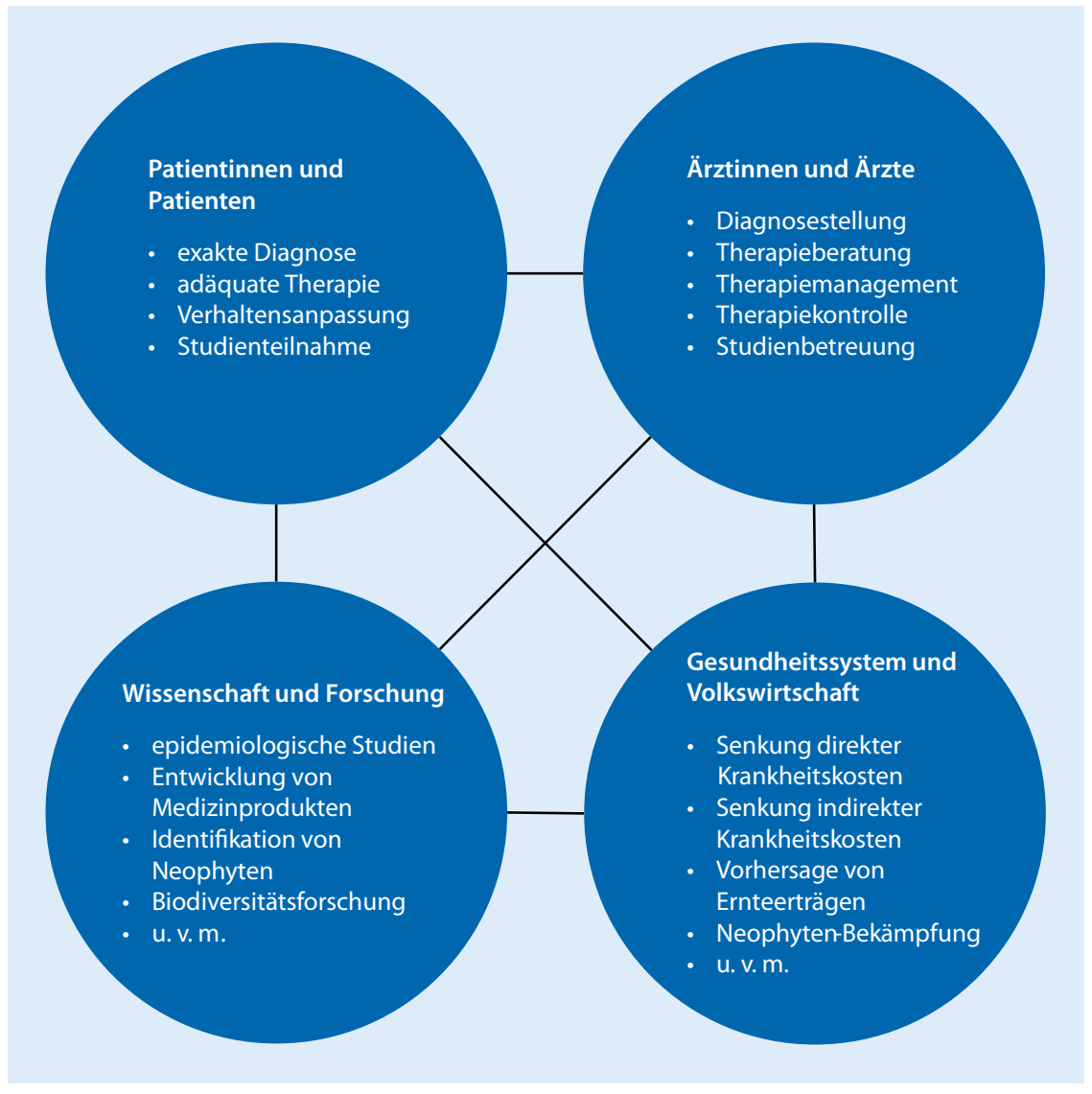

Abb. $1 \Delta$ Gesellschaftsgruppen bzw. -bereiche, die von Pollendaten profitieren. Die Gruppen bzw. Bereiche bedingen sich gegenseitig. So profitieren Patienten von optimalen Arbeitsbedingungen für Ärzte und durch adäquate Therapien und Verhaltensanpassungen lassen sich direkte und indirekte Krankheitskosten senken

geeigneter Analysten, die vertragsgemäß für die Stiftung arbeiten.

Die Pollen folgender Pflanzen werden derzeit standardmäßig gemessen: Hasel, Erle, Birke, Esche, Gräser, Roggen, Beifuß und Ambrosia [37]. Optional werden über 30 weitere Pollenarten erfasst.

Die Messungen erfolgen mittels Burkard-Fallen (Hirst-Typ-Fallen, siehe oben) nach der Richtlinie VDI 4252 Blatt 4 [2]. Aufgrund der mit der Falle verbundenen zeitintensiven manuellen Arbeitsschritte werden die Daten der Standardpollen mit einer Verzögerung von ein bis vier Tagen an den DWD zur Einarbeitung in die Pollenflugvorhersage weitergegeben (Details siehe unten). Außerdem werden die Daten ins Europäische Aeroallergennetzwerk eingespeist [38] und in Programme, deren Ziel die Korrelierung von Pollenlast und Beschwerden ist (Stichworte Pollentagebuch [39], Pollen App 4.0 [39], Allergie-
App „Husteblume“ [40]). Darüber hinaus bilden die Daten die Grundlage für den Pollenflugkalender (aktuell 4.0; [41]) und die Wochenpollenvorhersage der Stiftung [42] und sie werden für wissenschaftliche Zwecke zum Verkauf angeboten [43].

Weitere Arbeitsschwerpunkte der Stiftung umfassen die Aus- und Fortbildung von Pollenanalysten, die Ausrichtung von Pollensymposien sowohl auf nationaler als auch auf europäischer Ebene, die Beantwortung von Fragen aus Medizin und Öffentlichkeit, die Unterstützung von Adaptierungen an den Klimawandel und die Bearbeitung wissenschaftlicher Projekte, wie z.B. die Untersuchung der räumlichen Verteilung und Assoziation von Pollenkonzentration und polleninduzierten Beschwerden innerhalb einer Großstadt [44] und die Entwicklung von Techniken zur Messung der individuellen Pollenbelastung [45].
Für ihre Arbeiten steht der Stiftung ein jährliches Haushaltseinkommen von etwa $100.000 €$ zur Verfügung. Eine öffentliche Finanzierung erfolgt nicht.

\section{Pollenflugvorhersage durch den Deutschen Wetterdienst}

Der DWD, eine Bundesoberbehörde im Geschäftsbereich des Bundesministeriums für Verkehr und digitale Infrastruktur (BMVI), gibt gemäß seinem gesetzlichen Auftrag auf der Grundlage von (a) Wetterdaten, (b) phänologischen Daten, (c) Pollendaten der PID-Stiftung und weiterer Betreiber von Messstationen und (d) modellbasierten Pollenprognosen (Stichworte COSMO-ART, ICON-ART [46]) die Pollenflugvorhersage für Deutschland heraus (PollenflugGefahrenindex; [37]). Die Vorhersage steht der Bevölkerung über eine Webseite, einen Newsletter und per App kostenfrei zur Verfügung. Die Webseite gehört zu den am meisten abgerufenen Internetseiten des DWD (April 2016: 1,3 Mio. Abrufe). Neben der Pollenflugvorhersage bietet der DWD eine semiquantitative Pollenflugstatistik an [47].

\section{Pollenmessnetze anderer europäischer Staaten}

Die Pollenmessnetze Finnlands, Frankreichs, Großbritanniens, Österreichs und der Schweiz weisen untereinander und im Vergleich mit Deutschland sowohl Gemeinsamkeiten als auch Unterschiede auf. Unterschiede finden sich z.B. in der Finanzierung und der Verwaltung, der Anzahl der Messstellen, der Datenaufarbeitung und der Datenausgabe.

Die Schweiz ist der einzige der sechs oben genannten Staaten mit einem öffentlich finanzierten und öffentlich verwalteten Messnetz. Es ist beim Bundesamt für Meteorologie und Klimatologie der Schweiz, MeteoSchweiz, angesiedelt. In den anderen Staaten finden sich unterschiedliche Finanzierungs- und Verwaltungsstrukturen, ihnen gemeinsam ist jedoch die deutliche Unterfinanzierung der Messnetze. Frankreich ist das einzige der sechs Länder mit einer gesetzlichen Verpflichtung zur Messung luftgetragener biologischer Partikel, al- 
lerdings ohne Festlegung eines damit verbundenen Finanzbudgets.

Die Messstationen aller sechs Staaten arbeiten derzeit routinemäßig mit HirstTyp-Fallen (zur Austestung von Pollenvollautomaten in der Schweiz siehe unten). Vergleichbar mit Deutschland erfolgen die Probenaufbereitung und Auszählung der Pollen in Großbritannien und Österreich dezentral in jeder Messstation, während Finnland, Frankreich und die Schweiz zentral aufarbeiten und auszählen lassen. Als Gründe für die zentrale Aufarbeitung wurden die damit verbundene Kostenreduktion bzw. eine bessere Vergleichbarkeit der Daten genannt.

Vergleichbar mit Deutschland gehen die Daten in Finnland, Großbritannien und der Schweiz in eine numerische bzw. semiquantitative Pollenflugvorhersage ein. Im Gegensatz dazu geben Frankreich und Österreich ein sogenanntes Allergierisiko aus. In Frankreich gehen in die Berechnung des "Allergierisikos aufgrund von Pollenexposition“" neben Pollendaten, phänologischen Daten und Wetterdaten klinische Daten praktizierender Ärzte ein [48]. In Österreich werden für die Ausgabe des Allergierisikos neben Pollen- und Wetterdaten „chemische Wetterdaten wie Luftverschmutzungs- bzw. Luftqualitätsparameter (Ozon, Schwefeldioxid, Stickstoffdioxid und Feinstaub) berücksichtigt" [49].

Parallel $\mathrm{zu}$ ihrem Routinemessnetz mit Hirst-Typ-Fallen wird die Schweiz in den kommenden vier Jahren (2019-2022) den landesweiten Einsatz von Pollenvollautomaten (Rapid-E) testen. Der Einsatzphase gingen Testungen verschiedener Automatentypen und eine Studie zu den sozioökonomischen Vorteilen von Echtzeitpollendaten voraus [17].

\section{Gesetzliche Rahmenbedingungen in Deutschland}

Wären Pollen Luftverunreinigungen im Sinne des Bundes-Immissionsschutzgesetzes (BImSchG), müssten sie vergleichbar zu Luftverunreinigungen wie Feinstaub oder Stickstoffdioxid verpflichtend routinemäßig gemessen werden.
Zweck des BImSchG ist es, u. a. Menschen „vor schädlichen Umwelteinwirkungen zu schützen und dem Entstehen schädlicher Umwelteinwirkungen vorzubeugen“ (BImSchG, $\S 1$, Abs. 1; [50]). Schädliche Umwelteinwirkungen im Sinne des BImSchG sind unter anderem Luftverunreinigungen. Diese sind definiert als „Veränderungen der natürlichen Zusammensetzung der Luft, insbesondere durch ... Aerosole ...", wobei der Begriff „Aerosole“ Bioaerosole einschließt (BImSchG, $\S 3$, Abs. 4; [50]). Pollen sind Bioaerosole, allerdings ist der überwiegende Anteil der Pollenemissionen natürlichen Ursprungs und nicht anthropogen bedingt. Damit fallen Pollen eher nicht in den Geltungsbereich des BundesImmissionsschutzgesetzes mit der Folge, dass in Deutschland derzeit keine gesetzliche Verpflichtung zur Messung von Pollen besteht.

Eine gesetzliche Grundlage für die (verpflichtende) Messung von Pollen ist aber nicht zwingend erforderlich. Zum einen können sowohl der Bund als auch die Länder durch Rechtsverordnungen festlegen, dass über die im BundesImmissionsschutzgesetz bzw. die in der 39. Bundes-Immissionsschutzverordnung geregelten Stoffe hinaus weitere Stoffe in der Umgebungsluft gemessen werden müssen (Festlegung einer sogenannten Messverpflichtung). Zum anderen dürfen sowohl der Bund als auch die Länder auch ohne Rechtsverordnung messen (keine Sperrwirkung durch das Bundes-Immissionsschutzgesetz).

\section{Möglichkeiten eines verlässlichen bundesweiten Pollenmessnetzes}

\section{Zukunftsvorstellungen der Stiftung Deutscher Polleninformationsdienst}

Ihre zukünftigen Aufgaben sieht die Stiftung Deutscher Pollenformationsdienst (a) im Messen von Pollen, (b) im E-Health-Bereich, (c) in der Bearbeitung wissenschaftlicher Fragestellungen und (d) in der Öffentlichkeitsarbeit.

Das bestehende Pollenmessnetz zur Erfassung der Hintergrundbelastung sollte zukünftig aus mindestens 30 manuellen Pollenfallen ergänzt durch 5-7 Pollenvollautomaten bestehen ( $\mathrm{Hy}$ - bridmessnetz), die die Hintergrundbelastung für alle Regionen Deutschlands abbilden. Die Pollendaten sollten in Vorhersagemodelle (Stichwort SI$\mathrm{LAM}=$ „System for Integrated modeLling of Atmospheric coMposition" [51]) eingespeist werden. Ergänzend zur Messung der Hintergrundbelastung sollten Techniken zur Messung der individuellen Pollenbelastung weiterentwickelt werden [45].

Im E-Health-Bereich werden die Schwerpunkte auf der Zusammenführung von Pollendaten mit Symptomdaten, soziodemografischen Daten, Daten zur Luftqualität und evidenzbasierten individuellen Therapiehinweisen (Stichworte Pollen App 5.0, Pollen App 6.0), in der Förderung der Patientenselbsthilfe durch e-health-unterstützte Patientenaktivierung (siehe evidenzbasierte Ergebnisse für die Allergie-App „Husteblume " [52]) und in der Einbindung von Google-Daten in die Vorhersage pollenassoziierter Beschwerden und deren Therapie [53] liegen.

Zur Wahrnehmung dieser Aufgaben benötigt die Stiftung finanzielle Unterstützung über ihren aktuellen Haushalt hinaus. Insbesondere der Arbeitsbereich Pollenmessnetz kann mit den derzeitigen Mitteln weder adäquat fortgeführt noch weiterentwickelt werden. Dies beinhaltet zum einen die angemessene Bezahlung der Analysetätigkeit an den Messstellen und zum anderen die Erweiterung des bestehenden Messnetzes um eine entsprechende Anzahl an Pollenvollautomaten.

\section{Ideen des DWD zu einem bundes- weiten Automatenmessnetz}

Pollendaten sind eine der vier Säulen, auf denen die Pollenflugvorhersage des DWD aufgebaut ist (siehe oben). Vor dem Hintergrund der systembedingten geringen zeitlichen Auflösung und zeitlichen Verzögerung der Bereitstellung von Pollendaten unter Verwendung von HirstTyp-Fallen beteiligt sich der DWD seit Jahren an der Eignungsprüfung von Pollenvollautomaten. Aktuell erprobt er an seinem Standort Freiburg den Pollenvollautomaten Rapid-E (siehe oben). Sollte sich das Gerät hinsichtlich bestimmter Qualitätskriterien wie Reproduzierbarkeit, Präzision und Sensitivität als geeig- 
net für die routinemäßige Messung von Pollen erweisen, plant der DWD das Aufstellen von Rapid-E-Geräten an weiteren DWD-Standorten. Aktuelle Überlegungen priorisieren Hintergrundmessstellen im urbanen Raum, um eine möglichst große Anzahl Betroffener mit Pollenfluginformationen und -vorhersagen zu erreichen.

\section{Modellcharakter von ePIN}

Das Elektronische Polleninformationsnetzwerk Bayern wird derzeit in Bayern im Auftrag des Bayerischen Staatsministeriums für Gesundheit und Pflege (StMGP) durch das Bayerische Landesamt für Gesundheit und Lebensmittelsicherheit in Kooperation mit dem Bayerischen Landesamt für Umwelt (LfU) und mit Unterstützung durch das Zentrum Allergie und Umwelt, Technische Universität und Helmholtz Zentrum München, aufgebaut $[54,55]$. Die Besonderheiten von ePIN sind:

- weltweit erstmalig flächendeckender Einsatz von Pollenvollautomaten (BAA 500) für die Bereitstellung von Echtzeitpollendaten (8 Pollenvollautomaten, ergänzt durch 4 manuelle Pollenfallen; [54]),

- systematische Ermittlung der notwendigen Anzahl und Lokalisation der Messstellen [29, 55],

- öffentliche Finanzierung und öffentliche Verantwortlichkeit [54] und damit

- kostenfreie Verfügbarkeit der Pollendaten entsprechend dem Umweltinformationsgesetz [56].

Die Ziele von ePIN liegen (a) in der Verbesserung der Pollenflugvorhersagen des DWD und anderer meteorologischer Anbieter (keine eigenen Vorhersagen) durch Echtzeitpollendaten von systematisch ausgewählten, repräsentativen Messstandorten und (b) in der Förderung des wissenschaftlichen Erkenntnisgewinns im Bereich Allergologie, Aerobiologie und Klimatologie.

Aufgrund der Besonderheiten und Ziele von ePIN hat das Netzwerk Modellcharakter sowohl für das gesamte Bundesgebiet als auch über Deutschland hinaus.

\section{Diskussion}

\section{Kosten und Datenrepräsentativität}

Argumente gegen ein bundesweites Pollenmonitoring beziehen sich $u$. a. auf die Kostenfrage („Wer soll das bezahlen?") und auf Zweifel an der Repräsentativität der Daten („stimmen nicht mit meinen Beschwerden überein“).

Der Arbeitskreis kommt $\mathrm{zu}$ dem Schluss, dass die Kosten, die durch Unterhalt und Weiterentwicklung des Messnetzes entstünden, wesentlich niedriger wären als die Kosten, die dem Gesundheitssystem und der Gesellschaft durch pollenassoziierte allergische Erkrankungen entstehen. Bereits eine geringe Senkung der Krankheitskosten durch ein weiterentwickeltes Pollenmessnetz würde nach Meinung von Experten zu einem positiven Kosten-Nutzen-Verhältnis führen [17].

Mit Zweifeln an der Repräsentativität von Pollendaten haben sich Patienten zum Beispiel an den Deutschen Allergie- und Asthmabund gewandt. In epidemiologischen Studien lässt sich eine klare Assoziation von Pollenlast und Beschwerden zeigen [10-13]. Dennoch kann es auf individueller Ebene zu Abweichungen kommen. Sie können zum einen mit der derzeitigen Unterpräsens von Messstellen in bestimmten Regionen Deutschlands zusammenhängen (siehe oben). Zum anderen können z. B. variierende Allergengehalte von Pollen zu Diskrepanzen dieser Art führen [28, 57] oder auch lokalisierte Belastungsspitzen, die mit einem Hintergrundmessnetz nicht erfasst werden können (zur Entwicklung von Techniken zur Messung der individuellen Pollenbelastung siehe oben).

\section{Falsche Pollenflugvorhersage - na und?}

Falsche Pollenflugvorhersagen sowohl in die eine als auch in die andere Richtung können gesundheitliche Konsequenzen nach sich ziehen: Die Vorhersage zu hoher Pollenmengen kann zu einer Überdosierung von Medikamenten führen (siehe Tertiärprävention) und damit $\mathrm{zu}-$ vermeidbaren - Nebenwirkungen. Die
Vorhersage zu niedriger Pollenmengen kann gesundheitliche Beeinträchtigungen nach sich ziehen und zur Entwicklung eines sogenannten Etagenwechsels, dem Übergang von „nur" Heuschnupfen $\mathrm{zu}$ allergischem Asthma, beitragen [58].

\section{Weiterentwicklung von \\ Pollenmessnetz und Pollenflug- vorhersage: Standortoptimierung, Echtzeitmessungen, freie Datenverfügbarkeit}

Wesentlich für ein bundesweites Hintergrundmessnetz sind Anzahl und Lokalisation der Standorte. Sie sollen für eine gegebene Region repräsentativ sein, nicht redundant messen und müssen bestimmte Bedingungen hinsichtlich Aufstellhöhe, Mindestabstand zu lokalen Pollenemittenten etc. erfüllen. Das Vorgehen zur Ermittlung der Anzahl benötigter Messstellen im Rahmen von ePIN und die Richtlinie VDI 4252 Blatt 4 geben hierzu Informationen $[2,29,55]$.

Hinsichtlich der Messtechnik sieht der Arbeitskreis die Zukunft in einer Kombination von Pollenvollautomaten zur Optimierung der Pollenflugvorhersage und Hirst-Typ-Fallen zur Kontrolle und Optimierung der Pollenvollautomaten sowie zur Aufrechterhaltung der historischen Messreihen (Stichwort Klimawandel). Ein wichtiges Kriterium für die Festlegung auf einen Automatentypus ist die Fähigkeit zur Datenarchivierung für retrospektive Analysen (relevant für wissenschaftliche Fragestellungen).

Die kostenfreie Verfügbarkeit von Pollendaten, wie sie im Falle der Finanzierung der Messung mit öffentlichen Geldern gesetzlich vorgeschrieben ist [56], sorgt für Datentransparenz und gleiche Wettbewerbsbedingungen hinsichtlich der Erstellung von Pollenflugvorhersagen und macht das Arbeiten mit sogenannten Fake-Daten, d.h. fiktiven Werten, überflüssig [29].

\section{Weitere Zukunftsaspekte}

Weitere Stichpunkte hinsichtlich der Weiterentwicklung des Pollenmessnetzes und der Pollenflugvorhersagen sind derzeit z.B. 
- Ausbau und Optimierung von Symptomvorhersagen und Ausgabe individueller Therapieempfehlungen (Stichwort Schwellenwertbestimmung, siehe oben; weitere Programme siehe z.B. PASY$\mathrm{FO}=$ „Personal Allergy Symptom Forecasting System“ [59]),

- Allergenmonitoring, d.h. das Messen des Allergengehaltes von Pollen bzw. des Allergengehaltes der Luft $[34,57]$,

- Monitoring und Bewertung der Wechselwirkungen zwischen Allergenen und Luftverunreinigungen [49],

- personalisierte Pollenmessungen [45],

- Citizen Science, d.h. die Einbindung von Laien in die wissenschaftliche Arbeit (z. B. Identifikation von Neophyten, Phänologie; [60]), - Nutzung von Satellitendaten (Stichwort Copernicus [61]).

\section{Fazit}

Aufgrund der Bedeutung allergener Pollen für die menschliche Gesundheit und allergischer Erkrankungen für das Gesundheitssystem spricht sich der Arbeitskreis dafür aus, das bundesweite Pollenmonitoring in den Katalog staatlicher Aufgaben aufzunehmen, die der grundlegenden Versorgung der Bevölkerung mit wesentlichen Gütern und Dienstleistungen dienen (öffentliche Daseinsvorsorge).

Der Arbeitskreis leitet diese Empfehlung aus dem hohen Anteil der Bevölkerung $\mathrm{ab}$, der von allergenen Pollen direkt betroffen ist. Die Aufnahme des bundesweiten Pollenmonitorings in den Katalog staatlicher Aufgaben garantiert die nachhaltige Bereitstellung von transparenten und frei verfügbaren Pollendaten sowie die nachhaltige Weiterentwicklung des Messnetzes.

Der Arbeitskreis kommt zu dem Schluss, dass die Kosten, die dem Staat durch Unterhalt und Weiterentwicklung des Messnetzes entstünden, wesentlich niedriger wären als die Kosten, die dem Gesundheitssystem und der Gesellschaft durch pollenassoziierte allergische Erkrankungen entstehen. Bereits eine geringe Senkung der Krankheitskosten durch ein weiterentwickeltes Pollenmessnetz würde $\mathrm{zu}$ einem positiven Kosten-Nutzen-Verhältnis führen.

Hinsichtlich möglicher Zuständigkeiten im Rahmen der öffentlichen Daseinsvorsorge wurden im Arbeitskreis mehrere Lösungsansätze diskutiert. Dazu gehörte die Möglichkeit, eine Bundeseinrichtung, wie z.B. den DWD, mit der Fortführung und Weiterentwicklung des bundesweiten Pollenmessnetzes zu beauftragen. Eine andere Möglichkeit wäre, die Aufgabe an die Stiftung Deutscher Polleninformationsdienst oder andere Einrichtungen zu übertragen.

Unabhängig von der zukünftigen $\mathrm{Zu}$ ständigkeit kommt der Kooperation von messtechnischen, klinischen und wissenschaftlichen Einrichtungen eine grundsätzliche Bedeutung für die adäquate gesundheitliche Vorsorge zu.

\section{Korrespondenzadresse}

\section{Fachübergreifender Arbeitskreis} „Bundesweites Pollenmonitoring“ Berlin, Deutschland conny.hoeflich@uba.de

Fachgebiet II 1.5 „Umweltmedizin, gesundheitliche Bewertung", Umweltbundesamt, Corrensplatz 1, 14195 Berlin, Deutschland

Mitglieder des Fachübergreifenden Arbeitskreises „Bundesweites Pollenmonitoring“. Regine Baeker (Ministerium für Arbeit, Soziales, Gesundheit, Frauen und Familie des Landes Brandenburg, Referat 43, Öffentlicher Gesundheitsdienst, Infektionsschutz, Umwelthygiene, Zivil- und Katastrophenschutz); Prof. Dr. Karl-Christian Bergmann (Stiftung Deutscher Polleninformationsdienst; Deutsche Gesellschaft für Allergologie und klinische Immunologie); Prof. Dr. Jeroen Buters (Zentrum Allergie und Umwelt, Technische Universität München und Helmholtz Zentrum München; Deutsche Gesellschaft für Allergologie und klinische Immunologie); Thomas Dümmel (Freie Universität Berlin, Institut für Meteorologie); Dr. Christina Endler (Deutscher Wetterdienst, Referat Lufthygiene); Prof. Dr. Thomas Fuchs (Ärzteverband Deutscher Allergologen e. V.); Dr. Stefan Gilge (Deutscher Wetterdienst, Referat Lufthygiene); Kai Gloyna (Landesamt für Gesundheit und Soziales Mecklenburg-Vorpommern, Abteilung 3, Fachbereich Infektiologie); Ruth Heesen (Verein Deutscher Ingenieure, VDI/DIN-Kommission Reinhaltung der Luft (KRdL) - Normenausschuss); Prof. Dr. Caroline Herr (Gesellschaft für Hygiene, Umweltmedizin und Präventivmedizin); Dr. Martin Hicke (Länderarbeitsgruppe Umweltbezogener Gesundheitsschutz); Dr. Conny Höflich (Umweltbundesamt, Fachgebiet II 1.5, „Umweltmedizin und gesundheitliche Bewertung"; Korrespondenzautorin); Prof. Dr. Ludger Klimek (Ärzteverband Deutscher Allergologen e. V.); Susanne Kutzora (Bayerisches Landesamt für Gesundheit und Lebensmittelsicherheit, AP 2
Arbeits- und Umweltmedizin/Epidemiologie); Dr. Marcel Langner (Umweltbundesamt, Abteilung II 4, "Luft"; Bund/Länder-Arbeitsgemeinschaft Immissionsschutz, Ausschuss Luftqualität/Wirkungsfragen/ Verkehr); Dr. Hans-Guido Mücke (Umweltbundesamt, Fachgebiet II 1.5, „Umweltmedizin und gesundheitliche Bewertung“); Gudrun Petzold (Länderarbeitsgruppe Umweltbezogener Gesundheitsschutz); Dr. Silvia Pleschka (Deutscher Allergie- und Asthmabund e. V.); Dr. Stefani Röseler (Rheinisch-Westfälische Technische Hochschule Aachen, Klinik für Dermatologie und Allergologie); Anja Schwalfenberg (Deutscher Allergie- und Asthmabund e. V.); Sven Simon (Länderarbeitsgruppe Umweltbezogener Gesundheitsschutz); Dr. Wolfgang Straff (Umweltbundesamt, Fachgebiet II 1.5, „Umweltmedizin und gesundheitliche Bewertung"); Alisa Weber (Bayerisches Landesamt für Gesundheit und Lebensmittelsicherheit, AP 2 Arbeits- und Umweltmedizin/Epidemiologie; Gesellschaft für Hygiene, Umweltmedizin und Präventivmedizin); Barbora Werchan (Stiftung Deutscher Polleninformationsdienst); Matthias Werchan (Stiftung Deutscher Polleninformationsdienst)

Danksagung. Unser herzlicher Dank gilt den Gästen der Gesprächstage, ohne deren Hilfe die fachgerechte Diskussion bestimmter Themenschwerpunkte unmöglich gewesen wäre: Uwe Berger (Medizinische Universität Wien, Forschungsgruppe Aerobiologie und Polleninformation, Österreich), Yolanda Clewlow (Met Office, Großbritannien), Bernard Clot (MeteoSchweiz, Schweiz), Ute Dauert (Umweltbundesamt, Fachgebiet II 4.2, "Beurteilung der Luftqualität", Deutschland), Marc Röckinghausen (Fachhochschule für öffentliche Verwaltung Nordrhein-Westfalen, Deutschland), Jörg Rechenberg (Umweltbundesamt, Fachgebiet II 2.1, „Übergreifende Angelegenheiten Wasser und Boden"), Mikhail Sofiev (Finnisches Meteorologisches Institut, Finnland) und Michel Thibaudon (Französisches Aerobiologisches Netzwerk, Frankreich). Herzlichen Dank auch an Lorraine Kaiser (Umweltbundesamt, Fachgebiet II 1.5, „Umweltmedizin und gesundheitliche Bewertung ") für ihre tatkräftige Unterstützung bei Fragen zu DeutschEnglisch-Übersetzungen.

\section{Einhaltung ethischer Richtlinien}

Interessenkonflikt. Der Fachübergreifende Arbeitskreis „Bundesweites Pollenmonitoring“ gibt an, dass kein Interessenkonflikt besteht.

Für diesen Beitrag wurden von den Autoren keine Studien an Menschen oder Tieren durchgeführt. Für die aufgeführten Studien gelten die jeweils dort angegebenen ethischen Richtlinien.

Open Access. Dieser Artikel wird unter der Creative Commons Namensnennung 4.0 International Lizenz (http://creativecommons.org/licenses/by/4.0/deed. de) veröffentlicht, welche die Nutzung, Vervielfältigung, Bearbeitung, Verbreitung und Wiedergabe in jeglichem Medium und Format erlaubt, sofern Sie den/die ursprünglichen Autor(en) und die Quelle ordnungsgemäßnennen, einen Link zur Creative Commons Lizenz beifügen und angeben, ob Änderungen vorgenommen wurden. 


\section{Literatur}

1. Stiftung Deutscher Polleninformationsdienst (2016) Dringender Appell - Pollenfallen vor dem Aus. http://www. pollenstiftung.de/aktuelles-einzelansicht/ dringender-appell-pollenfallen-vor-dem-aus/ 9f632a97b8f2898056638cc6bfd549db/.Zugegriffen:21. Aug. 2018

2. Verein Deutscher Ingenieure (2019) Richtlinie VDI 4252 Blatt 4: Bioaerosole und biologische Agenzien - Ermittlung von Pollen und Sporen in der Außenluft unter Verwendung einer volumetrischen Methode für ein Messnetz zu allergologischen Zwecken. www.vdi.de/4252. Zugegriffen: 12. Febr. 2019

3. Bergmann KC, Heinrich J, Niemann H (2016) Current status of allergy prevalence in Germany: Position paper of the Environmental Medicine Commission of the Robert Koch Institute. Allergo J Int 25:6-10

4. Trautmann A, Kleine-Tebbe J (2013) Allergologie in Klinik und Praxis: Allergene, Diagnostik, Therapie. Thieme, Stuttgart

5. Langen U, Schmitz R, Steppuhn H (2013) Prevalence of allergic diseases in Germany. Results of the German Health Interview and Examination Survey for Adults (DEGS1). Bundesgesundheitsblatt Gesundheitsforschung Gesundheitsschutz 56(5/6):698-706

6. Schlaud M, Atzpodien K, Thierfelder W (2007) Allergic diseases. Results from the German Health Interview and Examination Survey for Children and Adolescents (KiGGS). Bundesgesundheitsblatt Gesundheitsforschung Gesundheitsschutz 50:701-710

7. Treudler R, Simon JC (2017) Pollen-related food allergy: An update. Allergo J Int 26:273-282

8. Haftenberger M, Laußmann D, Ellert U et al (2013) Prevalence of sensitisation to aeraoallergens and food allergens. Results of the German Health Interview and Examination Survey for Adults (DEGS1). Bundesgesundheitsblatt Gesundheitsforschung Gesundheitsschutz 56:687-697

9. Jäger S (2000) Ragweed (Ambrosia) sensitisation rates correlate with the amount of inhaled airborne pollen. A 14-year study in Vienna, Austria. Aerobiologia (Bologna) 16(1):149-153

10. Lake IR, Jones NR, Agnew M et al (2017) Climate change and future pollen allergy in Europe. Environ Health Perspect 125:385-391

11. Durham SR, Nelson HS, Nolte H et al (2014) Magnitude of efficacy measurements in grass allergy immunotherapy trials is highly dependent on pollen exposure. Arerugi 69:617-623

12. Gassner M, Gehrig R, Schmid-Grendelmeier $P$ (2013) Hay fever as a Christmas gift. N Engl J Med 368:393-394

13. Tosi A, Wüthrich B, Bonini M, Pietragalla-Köhler B (2011) Time lag between Ambrosia sensitisation and Ambrosia allergy:A20-yearstudy (1989-2008) in Legnano, Northern Italy. Swiss Med Wkly 141:w13253

14. Zuberbier T, Lötvall J, Simoens S, Subramanian SV, Church MK (2014) Economic burden of inadequate management of allergic diseases in the European Union: A GA(2) LEN review. Allergy 69(10):1275-1279

15. Schramm B, Ehlken B, Smala A, Quednau K, Berger K, Nowak D (2003) Cost of illness of atopic asthma and seasonal allergic rhinitis in Germany: 1-yr retrospective study. Eur Respir J 21:116-122

16. Bayerisches Landesamt für Gesundheit und Lebensmittelsicherheit (2015) Abschätzung der
Krankheitskosten der Pollenallergien für Bayern pro Jahr

17. Oberpriller $Q$, Vettori $A$, Iten $R$ (2017) Nutzen RealTime Pollendaten. INFRAS, Zürich

18. Taramarcaz P, Lambelet B, Clot B, Keimer C, Hauser $C$ (2005) Ragweed (Ambrosia) progression and its health risks: Will Switzerland resist this invasion? Swiss Med Wkly 135:37-38

19. Ziska L, Knowlton K, Rogers $C$ et al (2011) Recent warming by latitude associated with increased length of ragweed pollen season in central North America. Proc Natl Acad Sci U SA 108:4248-4251

20. Höflich C (2014) Klimawandel und Pollenassoziierte Allergien der Atemwege. Umw Mensch Informationsdienst 01:5-10

21. Durham OC (1946) The volumetric incidence of atmospheric allergens. IV. A proposed standard method of gravity sampling, counting, and volumetric interpolation of results. J Allergy (Cairo) 17:79-86

22. Werchan B, Werchan M, Mücke HG et al (2017) Spatial distribution of allergenic pollen through a large metropolitan area. Environ Monit Assess 189:169

23. Buters JTM, Antunes C, Galveias A et al (2018) Pollen and spore monitoring in the world. Clin Transl Allergy 8:9

24. Burkard Scientific Ltd (2018) Burkard 7 day recording volumetric spore sampler. http://www. burkardscientific.co.uk/agronomics/hirst_spore sampler.htm.Zugegriffen: 15. Okt. 2018

25. LANZONI srl (2018) VPPS ${ }^{\circledR}$ volumetric pollen and particle sampler. https://www.lanzoni.it/ campionatore-pollini.Zugegriffen: 15. Okt. 2018

26. Burkard Manufacturing Co. Ltd. (2018) Burkard Manufacturing Co. Ltd. Scientific equipment for medical, environmental, agricultural and laboratorysciences. http://burkard.co.uk/.Zugegriffen: 12. Nov. 2018

27. VDI/DIN-Kommission Reinhaltung der Luft (KRdL) - Normenausschuss (2019) DIN EN 16868. https://www.din.de/de/meta/suche/ 62730 !search?query $=16868$ (Zugriff: voraussichtlich ab Herbst 2019)

28. Buters J (2017) Pollen und Pollenwarndienste. In: Wichmann HE, Fromme H (Hrsg) Handbuch der Umweltmedizin. ecomed-Storck $\mathrm{GmbH}$, Landsberg am Lech (59. Erg. Lfg. 11/17)

29. Weber A (2017) Workshop „Pollenmonitoring Current Development". Umw Hyg Arbeitsmed 22:35-44

30. Oteros J, Pusch G, Weichenmeier I et al (2015) Automatic and online pollen monitoring. Int Arch Allergy Immunol 167:158-166

31. Helmut Hund GmbH (2018) Pollenmonitor.https:// www.hund.de/de/instrumente/pollenmonitore. html.Zugegriffen: 15. Okt. 2018

32. Plair SA (2018) Real-time airborne particle identifier. http://www.plair.ch/Technology.html. Zugegriffen: 15.Okt. 2018

33. Clot B (2018) New EUMETNET AutoPollen Programme. IAANewsI 83:3

34. Pfaar O, Calderon MA, Andrews CP et al (2017) Allergen exposure chambers: harmonizing current concepts and projecting the needs for the future-An EAACI Position Paper. Allergy 72:1035-1042

35. Stiftung Deutscher Polleninformationsdienst (2018) Stiftung - und ihre Aufgaben. http://www. pollenstiftung.de/stiftung/geschichte/. Zugegriffen:6. Okt. 2018

36. Stiftung Deutscher Polleninformationsdienst (2018) Pollenmessstationen in Deutschland. http://www.pollenstiftung.de/pollenvorhersage/ pollenmessstationen-in-deutschland/. Zugegriffen:21. Aug. 2018

37. Deutscher Wetterdienst (2018) Pollenflug-Gefahrenindex. https://www.dwd.de/DE/leistungen/ gefahrenindizespollen/gefahrenindexpollen. html.Zugegriffen:21. Aug. 2018

38. European Aeroallergen Network (2018) Willkommen zur Ean Datenbank. https://ean.polleninfo. eu/Ean/.Zugegriffen: 15. Okt. 2018

39. Stiftung Deutscher Polleninformationsdienst (2018) Pollentagebuch/Pollen-App. http://www. pollenstiftung.de/pollentagebuchpollen-app/. Zugegriffen: $15.0 \mathrm{kt} .2018$

40. Techniker Krankenkasse (2018) Husteblume - Die Allergie-App der Techniker. https://www.tk.de/ techniker/gesund-leben/digitale-gesundheit/ apps/husteblume-allergie-app-2025388. Zugegriffen: $15.0 k t .2018$

41. Werchan M, Werchan B, Bergmann KC (2018) German pollen calendar 4.0-Update based on 2011-2016 pollen data. Allergo J Int 27:69-71

42. Stiftung Deutscher Polleninformationsdienst (2018) Wochenpollenvorhersage. (Beispiel). http://www.pollenstiftung.de/aktuelleseinzelansicht/wochenpollenvorhersagemichelle-26092018/76e1391c7d1f7f35315470 e21 bdfef89/.Zugegriffen:5.Nov. 2018

43. Stiftung Deutscher Polleninformationsdienst (2018) Kosten Pollenflugdaten. http:// www.pollenstiftung.de/pollenvorhersage/ pollenflugdaten/.Zugegriffen: 15. Okt. 2018

44. Werchan B, Werchan M, Mücke H-G, Bergmann K-C (2018) Spatial distribution of pollen-induced symptoms within a large metropolitan area-Berlin, Germany. Aerobiologia (Bologna) 34(4):539-556

45. Werchan M, Sehlinger T, Goergen F, Bergmann KC (2018) The pollator: A personal pollen sampling device. Allergo J Int 27:1-3

46. Deutscher Wetterdienst (2018) ICON-ART und COSMO-ART.https://www.dwd.de/DE/forschung/ wettervorhersage/num_modellierung/03_ umweltvorhersage/icon_art_cosmo_art.html. Zugegriffen: 12.Nov. 2018

47. Deutscher Wetterdienst (2018) Pollenflugstatistik. https://www.dwd.de/DE/leistungen/pollen/ pollenstatistik.html?nn=16102. Zugegriffen: 15 . Okt. 2018

48. French Aerobiology Network (2018) Risks by pollen. http://www.pollens.fr/en/risks/risks-bypollen.php.Zugegriffen: 15. Okt. 2018

49. Medizinische Universität Wien (2018) Allergierisiko. https://www.pollenwarndienst.at/aktuellebelastung/allergierisiko.html. Zugegriffen: 15 . Okt. 2018

50. Bundesministerium der Justizund fürVerbraucherschutz (2018) Gesetz zum Schutz vor schädlichen Umwelteinwirkungen durch Luftverunreinigungen, Geräusche, Erschütterungen und ähnliche Vorgänge (Bundes-Immissionsschutzgesetz BImSchG). https://www.gesetze-im-internet.de/ bimschg/inhalts_bersicht.html. Zugegriffen: 15 . Okt. 2018

51. Finnisches Meteorologisches Institut (2014) SILAM v.5.5 - System for integrated modelling of atmospheric composition. http://silam.fmi.fi/. Zugegriffen: 12.Nov. 2018

52. Institut für Medizinische Biometrie und Statistik der Albert-Ludwigs-Universität Freiburg (2017) Nutzungsverhalten und Wirksamkeit der Smartphone Applikation "Husteblume“ der Techniker Krankenkasse (Evaluationsstudie der Universität Freiburg. Abschlussbericht) 
53. Karatzas K, Papamanolis L, Katsifarakis N, Riga M, Werchan B, Werchan M et al (2018) Google trends reflect allergic rhinitis symptoms related to birch and grass pollen seasons. Aerobiologia (Bologna) 34(4):437-444

54. Bayerisches Landesamt für Gesundheit und Lebensmittelsicherheit (2018) Elektronisches Polleninformationsnetzwerk Bayern (ePIN). https://www.Igl.bayern.de/ gesundheit/arbeitsplatz_umwelt/biologische umweltfaktoren/bioaerosole/epin.htm. Zugegriffen:21. Aug. 2018

55. Bayerisches Staatsministerium für Gesundheit und Pflege (2018) Abschlussbericht ePIN-HEALTH: Studie zum Aufbau eines elektronischen Polleninformationsnetzwerkes in Bayern (ePIN-HEALTH) (Im Auftrag des Bayerischen Staatsministeriums für Gesundheit und Pflege.)

56. Bundesministerium der Justiz und für Verbraucherschutz (2018) Umweltinformationsgesetz. https://www.gesetze-im-internet.de/uig_2005/. Zugegriffen: 16. Okt. 2018

57. Buters J, Prank M, Sofiev M et al (2015) Variation of the group 5 grass pollen allergen content of airborne pollen in relation to geographic location and time in season. J Allergy Clin Immunol 136:87-95

58. Bastl K, Berger M, Bergmann KC, Kmenta M, Berger U (2017) The medical and scientific responsibility of pollen information services. Wien Klin Wochenschr 129:70-74

59. European Centre for Medium-Range Weather Forecasts (2018) PASYFO_Personal Allergy Symptom Forecasting System. http://pasyfo.lt/ en/.Zugegriffen: 16.Okt. 2018

60. Wissenschaft im Dialog, Museum für Naturkunde (2018) Bürger schaffen Wissen. Die Citizen Science Plattform. https://www.buergerschaffenwissen. de/.Zugegriffen: 18.Okt. 2018

61. European Commission (2018) Copernicus Europe's eyes on Earth. http://copernicus.eu/. Zugegriffen: $16.0 \mathrm{kt} .2018$ 